\title{
Stabilizing polynomial approximation of explicit MPC
}

Michal Kvasnica, Johan Löfberg and Miroslav Fikar

\section{Linköping University Post Print}

N.B.: When citing this work, cite the original article.

Original Publication:

Michal Kvasnica, Johan Löfberg and Miroslav Fikar, Stabilizing polynomial approximation of explicit MPC, 2011, Automatica, (47), 10, 2292-2297.

http://dx.doi.org/10.1016/j.automatica.2011.08.023

Copyright: Elsevier

http://www.elsevier.com/

Postprint available at: Linköping University Electronic Press

http://urn.kb.se/resolve?urn=urn:nbn:se:liu:diva-71641 


\title{
Stabilizing Polynomial Approximation of Explicit MPC *
}

\author{
Michal Kvasnica $^{\mathrm{a}}$, Johan Löfberg ${ }^{\mathrm{b}}$, Miroslav Fikar ${ }^{\mathrm{a}}$ \\ ${ }^{a}$ Faculty of Chemical and Food Technology, Slovak University of Technology in Bratislava, 81237 Bratislava, Slovakia \\ ${ }^{\mathrm{b}}$ Division of Automatic Control, Department of Electrical Engineering, Linköping University, SE-581 83 Linköping, Sweden
}

\begin{abstract}
A given explicit piecewise affine representation of an MPC feedback law is approximated by a single polynomial, computed using linear programming. This polynomial state feedback control law guarantees closed-loop stability and constraint satisfaction. The polynomial feedback can be implemented in real time even on very simple devices with severe limitations on memory storage.
\end{abstract}

Key words: Asymptotic stability; Predictive control; Function approximation.

\section{Introduction}

In explicit Model Predictive Control [4], parametric programming [6] is used to construct a function $\mu$ which maps state measurements $x$ onto the optimal control inputs. Provided such a $\mu$ exists, real-time implementation of MPC in Receding Horizon fashion (RHMPC) boils down to a mere function evaluation. For a rich class of MPC setups, $\mu$ can be shown to be a piecewise affine (PWA) function defined over $N_{\mathcal{R}}$ polytopic regions. The main practical limitation, however, is that the number of regions grows quickly with problem size, having negative impact on the required memory storage and processing power. The number of regions of $\mu$ can be reduced e.g. by move blocking [24], by model reduction techniques [13], or by relaxing optimality [2]. Another direction is to a-posteriori simplify the regions either by merging $[10,17]$, by replacing them by hyperboxes [15] or by simplices [12]. Evaluation of $\mu$ for a particular value of $x$ can be simplified by organizing the regions into a binary search tree [23], or by building a lattice representation [25] of the PWA function $\mu$. A common denominator of all referenced approaches is that they lead to a simpler (sub)optimal RHMPC feedback $\tilde{\mu}$, which still is a PWA function. As a consequence, although a remarkable reduction of complexity can be achieved in certain

\footnotetext{
* This paper was not presented at any IFAC meeting. Corresponding author M. Kvasnica. Tel. +421-2-59325352. Fax +421-2-59325340.

Email addresses: michal.kvasnica@stuba.sk (Michal Kvasnica), johanl@isy.liu.se (Johan Löfberg), miroslav.fikar@stuba.sk (Miroslav Fikar).
}

cases, the memory footprint of the approximation $\tilde{\mu}$ still typically exceeds ten kilobytes. In this work we aim at simplify the RHMPC in such a way that it can easily be implemented on typical industrial hardware platforms, such as programmable logic controllers, which usually only provide $2-8 \mathrm{kB}$ of memory.

We propose to remove all regions of $\mu$ completely and to approximate it by a single polynomial feedback $\tilde{\mu}(x)$ of an a-priori fixed degree such that closed-loop stability and constraint satisfaction are preserved. The approach is applicable not only to linear systems, but also covers switched affine systems which belong to the class of hybrid systems [3]. Building upon our previous work $[16,19]$, the approximation is performed in two steps. First, a parameterization of a set of stabilizing controllers, referred to as the stability tube [8], is obtained using basic computational geometry tools. Subsequently we show how to search for the coefficients of $\tilde{\mu}$ by solving a single linear program (LP). If the LP is feasible, the polynomial control law is guaranteed to reside in the stability tube, and hence it is closed-loop stabilizing and satisfies constraints for all time.

The key advantage is that the memory footprint of the approximate feedback $\tilde{\mu}$ is minute compared to the storage of $\mu$. In particular, for the type of problems considered here, the total storage for $\tilde{\mu}$ is roughly equal to the footprint of a single region of $\mu$. It follows that the overall memory requirements are reduced $N_{\mathcal{R}}$ times. The price to be paid is the inherent loss of optimality. Moreover, certain assumptions on the shape of the stability tube have to be imposed in order to formulate the search for 
coefficients of $\tilde{\mu}$ as a single LP. Because of this, and since the LP-based search is based on sufficient conditions, it does not have to be always successful.

Compared to our previous work $[16,19]$, we report a detailed complexity analysis of the overall design procedure. A large case study is provided to illustrate how the computation scales with increasing problem size and to assess the overall success rate. More importantly, new ideas for reducing the size of the LP problem are presented and extend applicability of our approach to larger problems.

\section{Preliminaries}

The set of non-negative real numbers is denoted by $\mathbb{R}_{>0}$. Interior of a set $\Omega$ is int $(\Omega)$. We call a collection of polytopes $\left\{\mathcal{R}_{i}\right\}_{i=1}^{N_{\mathcal{R}}}$ the partition of a set $\Omega$ if $\Omega=\bigcup_{i=1}^{N_{\mathcal{R}}} \mathcal{R}_{i}$, and $\operatorname{int}\left(\mathcal{R}_{i}\right) \cap \operatorname{int}\left(\mathcal{R}_{j}\right)=\emptyset$ for all $i \neq j$. Each polytope $\mathcal{R}_{i}$ will be referred to as a region of the partition. A function $\mu: \mathbb{R}^{n_{x}} \rightarrow \mathbb{R}^{n_{u}}$ with domain $\Omega \subseteq \mathbb{R}^{n_{x}}$ is called Piecewise Affine (PWA) over polytopes if $\left\{\mathcal{R}_{i}\right\}_{i=1}^{N_{\mathcal{R}}}$ is a partition of $\Omega$ and $\mu(x):=K_{i} x+L_{i} \quad \forall x \in \mathcal{R}_{i}, i=1, \ldots, N_{\mathcal{R}}$.

We consider well-posed [3], stabilizable PWA systems in discrete time $x_{t+1}=f_{\mathrm{PWA}}\left(x_{t}, u_{t}\right)$, composed of finitely many local affine dynamics, each valid in a polytope $\mathcal{D}_{d} \subseteq \mathbb{R}^{n_{x}}$ :

$$
f_{\mathrm{PWA}}\left(x_{t}, u_{t}\right):=A_{d} x_{t}+B_{d} u_{t}+a_{d} \text { if } x_{t} \in \mathcal{D}_{d},
$$

where $x_{t} \in \mathbb{R}^{n_{x}}$ are the states and $u_{t} \in \mathbb{R}^{n_{u}}$ the inputs. The task is to control the PWA system (1) towards the origin (which is assumed to be an equilibrium of (1)) while fulfilling state and input constraints for all time, i.e. $x_{t} \in \mathcal{X}, u_{t} \in \mathcal{U}, \forall t \geq 0$, where $\mathcal{X} \subseteq \mathbb{R}^{n_{x}}$ and $\mathcal{U} \subseteq \mathbb{R}^{n_{u}}$ are assumed to be non-empty polytopic sets containing the origin in their respective interiors.

We define for the PWA system (1) the constrained finite time optimal control (CFTOC) problem

$$
\begin{aligned}
& J_{N}^{*}\left(x_{t}\right)= \min _{U_{N}} \ell_{N}\left(x_{t+N}\right)+\sum_{k=0}^{N-1} \ell\left(x_{t+k}, u_{t+k}\right) \\
& \text { s.t. }\left\{\begin{array}{l}
x_{t+k+1}=f_{\mathrm{PWA}}\left(x_{t+k}, u_{t+k}\right), \\
u_{t+k} \in \mathcal{U}, x_{t+k} \in \mathcal{X}, x_{t+N} \in \mathcal{X}_{f},
\end{array}\right.
\end{aligned}
$$

where $x_{t+k}$ is the future evolution of (1) over a prediction horizon $N$, given the initial condition $x_{t}$ and the vector of future control inputs $U_{N}:=\left[u_{t}^{T}, \ldots, u_{t+N-1}^{T}\right]^{T} . \mathcal{X}_{f} \subseteq \mathcal{X}$ is a polytopic terminal set with $\mathbb{0}_{n_{x}} \in \mathcal{X}_{f}, \ell_{N}\left(x_{t+N}\right)=$ $\left\|Q_{N} x_{t+N}\right\|_{p}$ is the terminal penalty, and $\ell\left(x_{t+k}, u_{t+k}\right)=$ $\left\|Q_{x} x_{t+k}\right\|_{p}+\left\|Q_{u} u_{t+k}\right\|_{p}$ is the stage cost. It is assumed that $p \in\{1, \infty\}$ in $(2 \mathrm{a})$. For problems of modest size it is possible to characterize the RHMPC feedback law $\mu: \Omega \rightarrow \mathcal{U}$ and the optimal value function $J_{N}^{*}: \Omega \rightarrow$ $\mathbb{R}_{\geq 0}$ explicitly as PWA functions of $x_{t}[4,6]$. Here, $\Omega:=$ $\left\{x_{t} \mid \exists u_{t}, \ldots, u_{t+N-1}\right.$ s.t. (2b) hold $\}$, and it is partitioned into $N_{\mathcal{R}}$ polytopic regions $\mathcal{R}_{i}$.

Assumption 2.1 The RHMPCfeedback $\mu\left(x_{t}\right)$ is closedloop stabilizing, feasible for all time [8] and a PWA Lyapunov function $V: \Omega \rightarrow \mathbb{R}_{\geq 0}$ for the closed-loop system $f^{C L}:=f_{P W A}\left(x_{t}, \mu\left(x_{t}\right)\right)$ exists $\forall x_{t} \in \Omega$ and is given.

This is not a restricting requirement but rather the aim of most (if not all) control strategies. We remark that if $N, Q_{x}, Q_{u}, Q_{N}, \mathcal{X}_{f}$ are chosen as in [1], then $\mu(\cdot)$ satisfies Assumption 2.1 and $V:=J_{N}^{*}$ is a Lyapunov function.

Theorem 2.2 ([20]) Let $\Omega$ be a bounded positively invariant set with $\mathbb{O}_{n_{x}} \in \operatorname{int}(\Omega)$ and let $\beta(\cdot)$ and $\bar{\beta}(\cdot)$ be $\mathcal{K}_{\infty}$-class functions. Then if there exists function $V$ : $\Omega \rightarrow \mathbb{R}_{\geq 0}$ with $V\left(\mathbb{O}_{n_{x}}\right)=0$, bounded by $\underline{\beta}(\|x\|) \leq V(x) \leq$ $\bar{\beta}(\|x\|)$, and satisfying $V\left(f^{C L}(x)\right) \leq \gamma V(x)$ for some $\gamma \in[0,1)$ and for all $x \in \Omega$, then the closed-loop system $f^{C L}$ is asymptotically stable in $\Omega$.

The freedom in $\gamma$ allows one to find a set of stabilizing controllers which render the function $V$ a control Lyapunov function. Such sets are denoted as stability tubes [8]:

$$
\begin{gathered}
\mathcal{S}(V, \gamma):=\left\{\left[\begin{array}{l}
x \\
u
\end{array}\right] \mid u \in \mathcal{U}, x \in \Omega, f(x, u) \in \Omega,\right. \\
V(f(x, u)) \leq \gamma V(x)\} .
\end{gathered}
$$

For the type of PWA systems (1), PWA Lyapunov functions $V$, and fixed $\gamma$, the tube can be computed explicitly using reachability analysis $[8$, Ch. 10.4] and represented as a (possibly non-convex) union of polytopes. To see this, note that for each feasible transition from region $\mathcal{R}_{i}$ to region $\mathcal{R}_{j}$ for which the value of $V$ decreases, (3) is a polytope $\mathcal{S}_{i, j}$ in the $x-u$ space. The whole stability tube is then given by $\mathcal{S}(V, \gamma):=\bigcup_{i=1}^{N_{\mathcal{R}}} \mathcal{S}_{i}$, where $\mathcal{S}_{i}:=\bigcup_{j=1}^{N_{\mathcal{R}}} \mathcal{S}_{i, j}, i=1, \ldots, N_{\mathcal{R}}$.

\section{Main Results}

We aim at approximating a given RHMPC control law $\mu$ by a single multivariate polynomial $\tilde{\mu}$ of pre-specified degree $\delta$ :

$$
\tilde{\mu}(x)=\alpha_{1} x+\alpha_{2} x^{2}+\cdots+\alpha_{\delta} x^{\delta} .
$$

Here, $\alpha_{i} \in \mathbb{R}^{n_{u} \times n_{x}}, i=1, \ldots, \delta$, are the coefficients to be determined, and $x^{i}$ is the element-wise $i$-th power of a vector $x \in \mathbb{R}^{n_{x}}$, i.e. $x^{i}=\left[x_{1}^{i}, x_{2}^{i}, \ldots, x_{n_{x}}^{i}\right]^{T}$. Note that in a multi-input case with $n_{u}>1$, (4) is a vectorvalued polynomial. The constant offset $\alpha_{0}$ is not considered in (4) since $\tilde{\mu}\left(\mathbb{O}_{n_{x}}\right)=\mathbb{O}_{n_{u}}$ must hold to attain stability. Formally, we aim at solving the following problem. 
Problem 3.1 Find the coefficients $\boldsymbol{\alpha}=\left\{\alpha_{1}, \ldots, \alpha_{\delta}\right\}$ of the polynomial state-feedback law (4) of fixed degree $\delta$ such that $\tilde{\mu} \approx \mu$ asymptotically stabilizes the PWA system (1) to the origin while fulfilling state and input constraints for all time.

To solve this problem, we exploit the inherent freedom of the Lyapunov function $V$, captured by its stability tube:

Theorem $3.2([8])$ Let the stability tube $\mathcal{S}(V, \gamma)$ be given. Then every control law $\tilde{\mu}(x)$ fulfilling $\left[\begin{array}{c}x \\ \tilde{\mu}(x)\end{array}\right] \in$ $\mathcal{S}(V, \gamma)$ asymptotically stabilizes the system $x^{+}=$ $f_{P W A}(x, \tilde{\mu}(x))$ for all $x \in \Omega$ to the origin.

Remark 3.3 The concept of stability tubes does not require that the function $V$ originates as a solution of the $M P C$ problem (2). In fact, the tube can be constructed for an arbitrary feedback law which admits a $P W A^{1}$ Lyapunov function on $\Omega$. It follows that the presented procedure can be applied to approximate arbitrary feedback laws with this property.

In the sequel we show that, given a stability tube $\mathcal{S}(V, \gamma)$, the polynomial $\tilde{\mu}$ satisfying $[\underset{\tilde{\mu}(x)}{x}] \in \mathcal{S}(V, \gamma), \forall x \in \Omega$ can be found by solving a single linear program under the following assumption:

Assumption 3.4 For a given Lyapunov function $V$ there exists a $\gamma \in[0,1)$ for which:

A1: a full-dimensional stability tube $\mathcal{S}(V, \gamma):=\bigcup_{i=1}^{N_{\mathcal{R}}} \mathcal{S}_{i}$ exists;

A2: for each $i=1, \ldots, N_{\mathcal{R}}$ either $\mathcal{S}_{i}:=\cup_{j} \mathcal{S}_{i, j}$ is a convex polytope, or an inner polytopic approximation $\mathcal{S}_{i} \subseteq \cup_{j} \mathcal{S}_{i, j}$ exists such that $\operatorname{proj}_{x} \mathcal{S}_{i}=\mathcal{R}_{i}$

A3: the union $\cup_{i} \mathcal{S}_{i}$ is connected.

Existence of $\mathcal{S}(V, \gamma)$ hints at existence of control laws, other than $\mu$, which would provide closed-loop stability and constraint satisfaction for all time. Connectivity is implied by the objective of approximating $\mu$ by a single continuous polynomial valid over the whole domain $\operatorname{dom}(\tilde{\mu})=\Omega$. Finally, convexity (and hence uniqueness) is dictated by the desire of being able to perform the approximation in a computationally efficient manner. If A2 does not hold, $\tilde{\mu}$ can still be found by solving a combinatorial problem. If $A 3$ is violated, the remedy would be to approximate independently each connected part of the tube, giving rise to a piecewise polynomial type of approximation.

Under this assumption, the tube consists of $N_{\mathcal{R}}$ polytopes in the state-input space:

$$
\mathcal{S}_{i}:=\left\{\left[\begin{array}{l}
x \\
u
\end{array}\right] \mid\left[\begin{array}{ll}
S_{i}^{x} & S_{i}^{u}
\end{array}\right]\left[\begin{array}{l}
x \\
u
\end{array}\right] \leq S_{i}^{0}\right\} .
$$

\footnotetext{
1 For piecewise quadratic Lyapunov functions the tube can no longer be represented as a union of polytopes, in general.
}

We remark that the whole tube $\mathcal{S}(V, \gamma):=\cup_{i} \mathcal{S}_{i}$ is not required to be convex. Define, for each $i=1, \ldots, N_{\mathcal{R}}$, a set of polynomials

$$
p_{i}(\boldsymbol{\alpha}, x):=S_{i}^{0}-S_{i}^{x} x-S_{i}^{u} \tilde{\mu}(x),
$$

where the cardinality of $p_{i}(\cdot)$ is equal to the number of constraints of the $i$-th element of the stability tube, i.e. the number of rows of $S_{i}^{0}$. Then we get the following straightforward result.

Lemma 3.5 Let a stability tube $\mathcal{S}(V, \gamma)$ satisfying Assumption 3.4 be given. If there exist coefficients $\alpha_{1}, \ldots, \alpha_{\delta}$ of $\tilde{\mu}$ as in (4) such that

$$
p_{i}(\boldsymbol{\alpha}, x) \geq 0, \forall x \in \mathcal{R}_{i}, i=1, \ldots, N_{\mathcal{R}},
$$

then $\tilde{\mu}$ solves Problem 3.1 .

Proof. First note that (7) with $p_{i}(\boldsymbol{\alpha}, x)$ as in (6) is equivalent, for a fixed $i$, to (5) with $u=\tilde{\mu}(x)$. Therefore if (7) admits a solution, then $[\tilde{\mu}(x)] \in \mathcal{S}_{i} \forall x \in \mathcal{R}_{i}$. Hence if $(7)$ holds for all $i=1, \ldots, N_{\mathcal{R}}$, it follows from Theorem 3.2 that $\tilde{\mu}$ provides closed-loop stability and constraint satisfaction for all $x \in \Omega$.

Lemma 3.5 suggests that finding $\tilde{\mu}$ of the form (4) as a solution to Problem 3.1 can be cast as finding the coefficients $\alpha_{1}, \ldots, \alpha_{\delta}$ such that polynomials $p_{i}(\boldsymbol{\alpha}, x)$ are non-negative over corresponding regions. There is a subtle, yet very important issue which makes solving problem (7) far from straightforward: even for a fixed $i$, all polynomials $p_{i}(\cdot)$ associated to region $\mathcal{R}_{i}$ must be nonnegative for all points $x \in \mathcal{R}_{i}$, not just for some of them (e.g. for the vertices of $\mathcal{R}_{i}$ ). One approach is to employ the Positivstellensatz and show positivity of polynomials by solving a sum-of-squares problem, as suggested in [16]. However, as documented in [19], such a procedure is, from a practical point of view, limited to smallscale problems only. A different direction is therefore persuaded here, which is based on the following theorem, originally formulated by Pólya [11] to show strict positivity of polynomials and later extended to the nonstrict case by [22]:

Theorem 3.6 (Pólya's theorem) If a homogeneous polynomial $p_{i}(\boldsymbol{\alpha}, x)$ is non-negative over a unit simplex, then all the coefficients of the extended polynomial $p_{i}^{M}(\boldsymbol{\alpha}, x)=p_{i}(\boldsymbol{\alpha}, x) \cdot\left(\sum_{j=1}^{n_{x}} x_{j}\right)^{M}$ are non-negative for a sufficiently large Pólya degree $M$.

Remark 3.7 Search for the coefficients $\boldsymbol{\alpha}$, such that $p_{i}^{M}(\boldsymbol{\alpha}, x)$ is non-negative over a simplex can be performed by using the more obvious reverse of Pólya's theorem, i.e. that non-negative coefficients of the extended polynomial imply its non-negativity over the whole simplex.

Corollary 3.8 Given a symbolic representation of coefficients of $p_{i}^{M}(\boldsymbol{\alpha}, x)$, the coefficients $\boldsymbol{\alpha}$ of $\tilde{\mu}$ can be found 
by solving a linear program. To see this, observe that $\boldsymbol{\alpha}$ enters (6) in a linear fashion per definition of $\tilde{\mu}$ as in (4). All constraints in (7) are therefore linear in $\boldsymbol{\alpha}$.

Notice, however, that Theorem 3.6 is not directly applicable to find $\boldsymbol{\alpha}$ from (7) as $\mathcal{R}_{i}$ are not unit simplices with $\mathbb{O}_{n_{r}} \in \mathcal{R}_{i}$, in general. Therefore we propose to represent the polytopic regions in their equivalent vertex representation, i.e. by

$$
\begin{aligned}
& \mathcal{R}_{i}=\left\{x \mid x=\sum_{j=1}^{\left|\mathcal{V}_{i}\right|} \lambda_{j}\left[\mathcal{V}_{i}\right]_{j}, \lambda \in \Lambda_{i}\right\}, \\
& \Lambda_{i}=\left\{\lambda \mid 0 \leq \lambda_{j} \leq 1, \sum_{j=1}^{\left|\mathcal{V}_{i}\right|} \lambda_{j}=1\right\} .
\end{aligned}
$$

Here, $\mathcal{V}_{i}$ are the vertices the $i$-th region, $\left|\mathcal{V}_{i}\right|$ denotes their cardinality, $\left[\mathcal{V}_{i}\right]_{j}$ is the $j$-th vertex of $\mathcal{R}_{i}$, and $\lambda=\left[\lambda_{1}, \ldots, \lambda_{\left|\mathcal{V}_{i}\right|}\right]$. By substituting for $x=\sum_{j} \lambda_{j}\left[\mathcal{V}_{i}\right]_{j}$ into (6) and (7) we get

$$
p_{i}(\boldsymbol{\alpha}, \lambda) \geq 0, \quad \forall \lambda \in \Lambda_{i}, i=1, \ldots, N_{\mathcal{R}}
$$

Notice that $\Lambda_{i}$ in $(9)$ are now $\left|\mathcal{V}_{i}\right|$-dimensional unit simplices and Theorem 3.6 can therefore be applied to find $\boldsymbol{\alpha}$ such that $p_{i}(\boldsymbol{\alpha}, \lambda)$ is non-negative $\forall \lambda \in \Lambda_{i}$, $i=1, \ldots, N_{\mathcal{R}}$. Also note that such change of variables is needed even if all $\mathcal{R}_{i}$ originally were simplices, since the Pólya's Theorem only applies if $\mathbb{O}_{n_{x}} \in \mathcal{R}_{i}$.

We can now state the main result of the paper, which is Theorem 3.9 and Algorithm 1 for calculating values of the coefficients $\alpha_{1}, \ldots, \alpha_{\delta}$ of the polynomial feedback law $\tilde{\mu}$ which is an admissible solution to Problem 3.1.

Theorem 3.9 Let the input arguments of Algorithm 1 satisfy Assumption 2.1 and assume that the tube $\mathcal{S}(V, \gamma)$ computed in Step 1 satisfies Assumption 3.4. If the LP (10) is feasible, the polynomial feedback law $\tilde{\mu}$ of the form (4) calculated by Algorithm 1 is a solution to Problem 3.1.

Proof. If (10) is feasible, then, according to Theorem 3.6, polynomials $p_{i}(\boldsymbol{\alpha}, \lambda)$ are non-negative over corresponding regions $\mathcal{R}_{i}$. This in turn implies that (7) is satisfied, which, according to Lemma 3.5, shows that $\tilde{\mu}(x)$ belongs to the stability tube $\mathcal{S}(V, \gamma), \forall x \in \Omega$. Therefore, by Theorem 3.2, $\tilde{\mu}$ is guaranteed to be closedloop stabilizing and feasible for all time.

Remark 3.10 Algorithm 1 is a non-iterative procedure and therefore it always terminates in a single pass, provided that all of its steps are successful. However, since Theorems 3.2 and 3.6 are only sufficient conditions for existence of a stabilizing feedback $\tilde{\mu}$, the algorithm may fail to find it even if one exists.

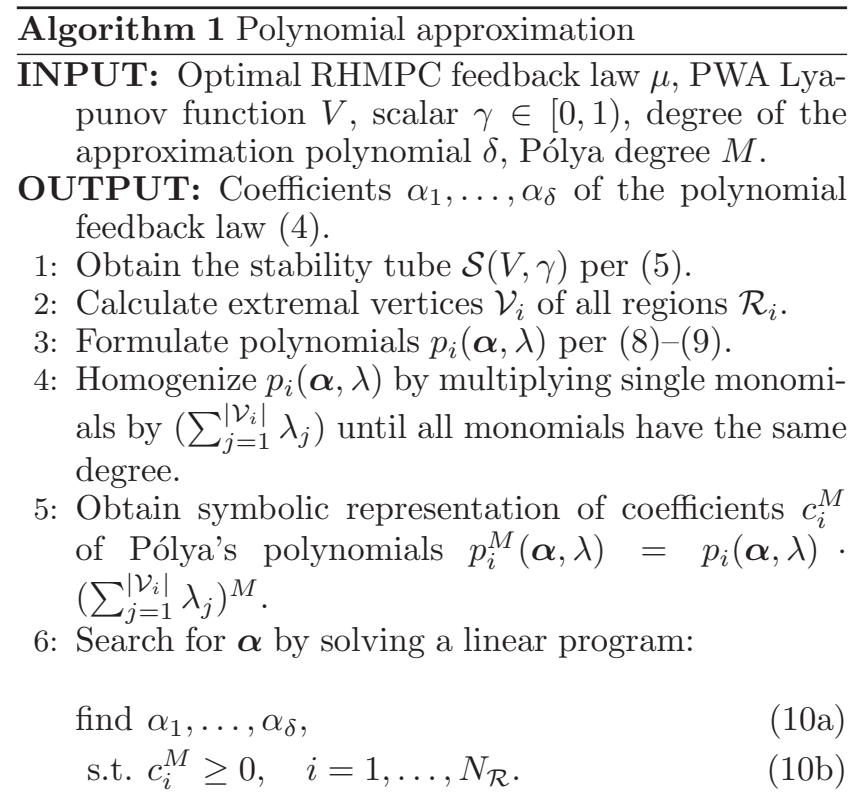

Instead of a pure feasibility objective in (10a), an alternative is to minimize the point-wise distance $\| \mu\left(x_{j}\right)-$ $\tilde{\mu}(x)\left(x_{j}\right) \|_{q}$ with $q \in\{1, \infty\}$ over some points $x_{j}$ (e.g. over the vertices of each $\left.\mathcal{R}_{i}\right)$. Doing so will let $\tilde{\mu}$ to follow the shape of $\mu(x)$ more tightly, hence mitigating the induced loss of optimality. Another approach is to aim for low-order polynomials. This can be done in three ways: (i) minimize the $\ell_{1}$ norm of $\boldsymbol{\alpha}$, which tends to give sparse solutions; (ii) use bisection in conjunction with Algorithm 1; or (iii) minimize the number of non-zero coefficients to a global minimum by solving a mixed-integer version of (10).

Example 3.11 Consider the following open-loop unstable PWA system [16]:

$$
x_{t+1}=\left\{\begin{aligned}
6 / 5 x_{t}-2 u_{t} & \text { if } x_{t} \geq 0 \\
-4 / 5 x_{t}+u_{t} & \text { otherwise }
\end{aligned}\right.
$$

with $u_{t} \in[-1,1]$ and $x_{t} \in[-4,4]$. With the choice of $p=1, Q_{x}=1, Q_{u}=1, N=\infty$ in (2) we obtain a stabilizing feedback $\mu$ and a Lyapunov function $J_{N}^{*}$ defined over 6 regions. The stability tube $\mathcal{S}(V, \gamma)$ for $V:=J_{N}^{*}$ and $\gamma=0.7$, the optimal RHMPC feedback $\mu\left(x_{t}\right)$, and its polynomial approximations $\tilde{\mu}$ of degrees $\delta=3,5,7$ computed by Algorithm 1 are shown in Figure 1.

\section{Complexity Analysis}

\subsection{Complexity of Algorithm 1}

Computation of stability tubes $\mathcal{S}(V, \gamma)$ in Step 1 can be done in $\mathcal{O}\left(N_{\mathcal{R}}^{2}\right)$ time, since all possible transitions 


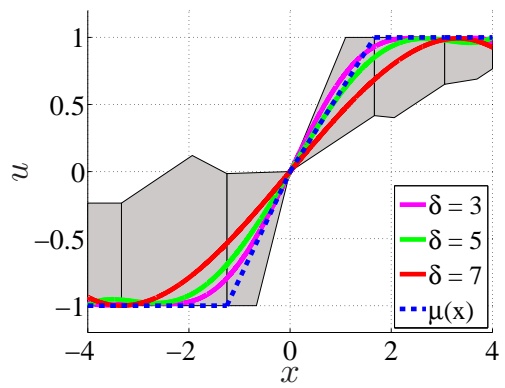

Fig. 1. Stability tube $\mathcal{S}(V, \gamma)$ for $\gamma=0.7$ (gray sets), optimal control law $\mu$ (blue dashed line), and stabilizing polynomials $\tilde{\mu}$ of different degrees $\delta$.

between various regions have to be investigated. The LP in Step 6 has $n_{u} n_{x} \delta$ variables (the coefficients $\alpha_{1}, \ldots, \alpha_{\delta}$ ) and $\mathcal{O}\left(N_{c}\right)$ constraints. Here, $N_{c}=\sum_{i=1}^{N_{\mathcal{R}}} N_{c, i}$ is the total number of coefficients of Pólya polynomials $p_{i}^{M}(\boldsymbol{\alpha}, \lambda)$, with $N_{c, i}=\left(\begin{array}{c}\delta_{p}+\left|\mathcal{V}_{i}\right|-1 \\ \delta_{p}\end{array}\right)$ and $\delta_{p}=\delta+M$, where $\left|\mathcal{V}_{i}\right|$ is the number of vertices of the $i$-th region.

Remark 4.1 The number of Pólya's coefficients, and hence the number of constraints of the LP (10), grows quickly with the number of states $n_{x}$. In the most general case, $\left|\mathcal{V}_{i}\right|=\mathcal{O}\left(2^{n_{x}}\right)$. This is the main bottleneck of the presented procedure. One way to mitigate such a quick growth is to triangulate the regions $\mathcal{R}_{i}$. Although this will increase the total number of regions to, at most, $\mathcal{O}\left(\sum_{i=1}^{N_{\mathcal{R}}}\left|\mathcal{V}_{i}\right|^{\left\lceil n_{x} / 2\right\rceil}\right)$, the gained advantage is that $\left|\mathcal{V}_{i}\right|$ stays fixed at $n_{x}+1, \forall i$. From numerical experiments we have observed that employing triangulation reduces the total number of constraints in (10) by a factor of 5 , on average.

Remark 4.2 Another option to reduce the size of the linear program (10) is to eliminate the redundant constraints. Full redundancy elimination would require solving $\mathcal{O}\left(N_{c}\right)$ copies of the LP (10), which clearly is not an option. Notice, however, that a valid solution to (10) has to be non-negative due to Theorem 3.6 and Remark 3.7. Therefore eliminating from (10b) those constraints where all constant multipliers in $c_{i}^{M}$ are non-negative will not affect feasibility. Numerical examples suggest that such a simple elimination reduces the number of constraints of (10) by a factor of 2 , on average.

\subsection{On-Line Complexity}

Implementing $\tilde{\mu}$ in a feedback arrangement reduces to a mere evaluation of the polynomial for a given $x$. Since the polynomial continuously covers the whole state-space of interest, no region search is necessary. Using Horner's scheme [9], $\tilde{\mu}$ can be evaluated ${ }^{2}$ by at most $1 / 2 n_{u} n_{x}(3 \delta+$

${ }^{2}$ If only fixed-point arithmetics is available, evaluation can be done as in [7]
5) FLOPS. Storing the coefficients $\alpha_{1}, \ldots, \alpha_{\delta}$ consumes $\delta n_{u} n_{x}$ floating point numbers. On the other hand, evaluating the optimal feedback law $\mu$ via a binary search tree [23] requires $\mathcal{O}\left(\log _{2} N_{\mathcal{R}}\right)$ FLOPS and the tree consumes $\mathcal{O}\left(N_{\mathcal{R}}\left(n_{x}+n_{u}\right)\right)$ memory elements. Complexity of the lattice representation [25], both in terms of runtime and memory, is $\mathcal{O}\left(N_{\mathcal{U}}^{2}\right)$ where $N_{\mathcal{U}}$ denotes the number of unique feedback laws.

\section{$5 \quad$ Examples}

\subsection{Standard PWA Benchmark}

Consider the following PWA system with 2 states and one input, introduced in $[3]: x^{+}=\left[\begin{array}{rr}\cos \theta & -\sin \theta \\ \sin \theta & \cos \theta\end{array}\right] x+\left[\begin{array}{l}0 \\ 1\end{array}\right] u$, where the value of $\theta$ switches depending on the value of the first element of the state vector: $\theta=-\pi / 3$ if $x_{1} \leq 0$, and $\theta=\pi / 3$ otherwise. State constraints $\left|x_{i}\right| \leq 5, i=1,2$ are assumed, along with input bounds $|u| \leq 1$. Even though the system is open-loop stable, a controller is needed to guarantee constraint satisfaction for all time. The explicit RHMPC feedback law $\mu$ was constructed by solving (2) with $Q_{x}=\mathbb{1}, Q_{u}=1, p=\infty$ and $N=\infty$, and consists of 112 regions shown in Figure 2(a). We have then applied Algorithm 1 to find approximate feedbacks $\tilde{\mu}$ of degrees $\delta=1, \ldots, 7$. The stability tube in Step 1 was computed for $\gamma=0.99$ and it satisfied Assumption 3.4. The polynomial of degree 7 is shown in Figure 2(b). To assess the induced loss of optimality, we have analyzed closed-loop profiles of states and inputs. The performance degradation is given by $\Delta_{J}:=\left(J^{*}-\tilde{J}\right) / J^{*}$, where $J^{*}$ is the value of (2a) for a closed-loop profile obtained by applying the optimal feedback $\mu(x)$, while $\tilde{J}$ is the cost of the closed-loop evolution driven by $u=\tilde{\mu}(x)$. The average values of $\Delta_{J}$ over 1000 equidistantly spaced initial conditions are reported in Table 1.

Table 1

Performance degradation for various degrees of the polynomial approximation $\tilde{\mu}$.

\begin{tabular}{|c||c|c|c|c|c|c|c|}
\hline$\delta$ & 1 & 2 & 3 & 4 & 5 & 6 & 7 \\
\hline$\Delta_{J}[\%]$ & 48 & 46 & 43 & 37 & 36 & 28 & 28 \\
\hline
\end{tabular}

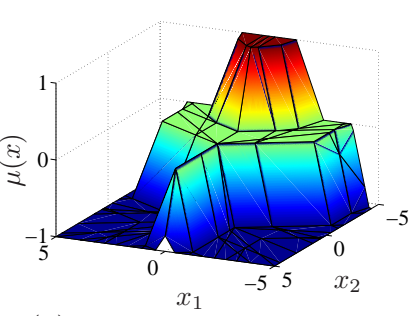

(a) RHMPC feedback $\mu$.

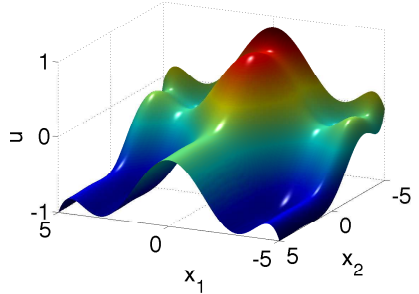

(b) Polynomial $\tilde{\mu}$ of degree 7 .
Fig. 2. Optimal RHMPC feedback law and its approximation. 


\subsection{Linear System}

Consider the following linear system with 4 states and 1 input:

$$
x^{+}=\left[\begin{array}{rrrr}
0.7 & -0.1 & 0.0 & 0.0 \\
0.2 & -0.5 & 0.1 & 0.0 \\
0.0 & 0.1 & 0.1 & 0.0 \\
0.5 & 0.0 & 0.5 & 0.5
\end{array}\right] x+\left[\begin{array}{l}
0.1 \\
1.0 \\
0.0 \\
0.0
\end{array}\right] u,
$$

which is subject to constraints $\left|x_{i}\right| \leq 5, i=1, \ldots 4$, and $|u| \leq 5$. The optimal RHMPC feedback law for $Q_{x}=10 \cdot \mathbb{1}_{4}, Q_{u}=0.1$, and $N=3$ has 230 regions in $4 \mathrm{D}$ state space. The lowest feasible degree of $\tilde{\mu}$ was $\delta=1$, leading to the linear feedback $\tilde{\mu}(x)=-0.0715 x_{1}$. It follows that while the on-line implementation of the optimal RHMPC controller would require the storage of 9290 floating point numbers (8140 for describing the 230 regions, and 1628 for the associated feedback laws), the polynomial feedback requires storing exactly one floating point number at the price of a $35 \%$ worst-case drop of performance.

This case also illustrates practical consequences of Remarks 4.1 and 4.2. Without any of them applied, the LP (10) for $\delta=3$ would have $5.8 \cdot 10^{6}$ constraints, which is above the limit of most LP solvers ${ }^{3}$. Performing triangulation per Remark 4.1 led to $1.8 \cdot 10^{6}$ inequalities. Further elimination of trivially redundant constraints per Remark 4.2 decreased this figure to $0.9 \cdot 10^{6}$.

\subsection{Random Systems}

To assess versatility of the presented approach, we have analyzed random PWA systems with 2 dynamics under state constraints $|x| \leq 5$ and input bounds $|u| \leq 1$. Three batches of random systems of various dimensions were considered, with 100 systems in each batch. For each system the optimal RHMPC feedback law $\mu$ was computed $^{4}$ by solving (2) with $Q_{x}=\mathbb{1}_{n_{x}}, Q_{u}=\mathbb{1}_{n_{u}}$, and $N=5 . Q_{N}$ and $\mathcal{X}_{f}$ were designed as in [1]. Subsequently, the stability tubes $\mathcal{S}(V, \gamma)$ were constructed for $V:=J_{N}^{*}$ and $\gamma=0.99$. In $62 \%$ of the 300 investigated problems the respective stability tubes satisfied Assumption 3.4. Important to notice is that the success rate was $97 \%$ when investigating a supplemental batch of 100 random linear systems.

The tubes were then triangulated according to Remark 4.1 and further processed by Algorithm 1. The runtime of triangulation never exceeded 20 seconds for any of the investigated examples. Enumeration of vertices in Step 2 never took more than 1 second using MPT [18]. The LP in Step 6 was formulated by

\footnotetext{
3 For instance, the 32-bit version of CPLEX only allows around $2 \cdot 10^{6}$ constraints.

${ }^{4} \mathrm{On}$ a $2.5 \mathrm{GHz} \mathrm{CPU}$ and $2 \mathrm{~GB}$ of RAM using Matlab R2009a and MPT 2.6.3.
}

Table 2

Data for random systems.

\begin{tabular}{|c||c|c|c|c|c|c|}
\hline$n_{x} / n_{u}$ & $N_{\mathcal{R}}$ & $N_{\mathcal{T}}$ & Step 1 & Step 6 & $\delta_{\text {min }}$ & $\Delta_{J}$ \\
\hline \hline \multirow{5}{*}{$2 / 1$} & 117 & 215 & 28 secs & 1 sec & 1 & $77 \%$ \\
\cline { 2 - 7 } & 180 & 329 & 54 secs & 2 secs & 5 & $23 \%$ \\
\cline { 2 - 7 } & 251 & 485 & 103 secs & 1 sec & 3 & $51 \%$ \\
\cline { 2 - 7 } & 288 & 552 & 152 secs & 2 secs & 6 & $37 \%$ \\
\hline \hline \multirow{3}{*}{$2 / 2$} & 115 & 206 & 62 secs & 3 secs & 2 & $81 \%$ \\
\cline { 2 - 7 } & 197 & 379 & 135 secs & 1 sec & 1 & $65 \%$ \\
\cline { 2 - 7 } & 277 & 522 & 164 secs & 1 sec & 5 & $35 \%$ \\
\cline { 2 - 7 } & 376 & 738 & 328 secs & 7 secs & 4 & $42 \%$ \\
\hline \hline \multirow{3}{*}{$3 / 1$} & 270 & 1258 & 293 secs & 3 secs & 3 & $12 \%$ \\
\cline { 2 - 7 } & 450 & 1755 & 551 secs & 1 sec & 1 & $72 \%$ \\
\cline { 2 - 7 } & 606 & 2963 & 829 secs & 12 secs & 6 & $41 \%$ \\
\cline { 2 - 7 } & 834 & 4278 & 1453 secs & 3 secs & 4 & $65 \%$ \\
\hline
\end{tabular}

YALMIP [21] and solved by CPLEX 12.1 [14]. Only degrees up to 7 were investigated due to practical reasons. The success rate of the LP-based procedure was $81 \%$. No obvious correlation between the number of regions of $\mu$ and the required degree $\delta$ in (10) was observed. Around $25 \%$ of all feasible cases admitted the existence of a linear approximation $\tilde{\mu}$, regardless of $n_{x}$ and $n_{u}$. Higher order approximations with minimal feasible degrees $\delta=2, \ldots, 6$ appeared with a roughly equal distribution.

A representative selection of the results is reported in Table 2 which shows how the computation scales with increasing problem size. Columns of the tables denote, respectively, state and input dimensions, number of regions $N_{\mathcal{R}}$, number of triangulated regions $N_{\mathcal{T}}$, runtime of construction of the stability tube in Step 1, runtime of the LP in Step 6 , minimum degree $\delta_{\min }$ for which the LP was feasible, and the average performance degradation induced by using $\tilde{\mu}$ of the minimal degree. Even though the average performance drop $\Delta_{J}$ might sound large, one has to take into account three facts. First, as discussed previously, performance usually improves if $\delta$ is enlarged. Second, and more importantly, design of any stabilizing feedback controller for PWA systems is a nontrivial task, even putting optimality aside. Finally, magnitudes of the reported performance drops are similar to what can be achieved by other techniques, see e.g. [12,5].

\section{Conclusions}

We have presented a novel way of deriving simple stabilizing feedback laws for the class of constrained linear and PWA systems. Stability and feasibility of the approximate polynomial controllers are guaranteed by employing the concept of stability tubes, which can be viewed as a parameterization of stabilizing feedback 
laws. It was illustrated that coefficients of the polynomials can be found by solving a single linear program. Triangulation and a cheap redundancy elimination were proposed as a way to significantly mitigate the size of the LP, hence allowing to process even large problems. Although the presented procedure inherently induces sub-optimality, the synthesized polynomial feedback not only guarantees stability and constraint satisfaction, but also puts very low requirements on its implementation in real time.

Certain restrictions have to be imposed on the shape of stability tubes in order to be able to find the approximation by solving a single LP. Investigation of a large number of random cases showed that a suitable tube was found in $60 \%$ of PWA systems, while the success rate is close to $100 \%$ when considering linear systems. If the tube has "unfavorable" shape, one would need to resort to a piecewise polynomial nature of the approximation. Although no obvious correlation between the number of elements of the tube and the degree of the approximate polynomial was observed, it can not be ruled out that higher order polynomials might be necessary to approximate more complex tubes.

\section{Acknowledgments}

The authors are pleased to acknowledge the financial support of the Scientific Grant Agency of the Slovak Republic under the grants 1/0079/09 and 1/0095/11 and of the Slovak Research and Development Agency under the contracts No. VV-0029-07 and No. LPP-0092-07.

\section{References}

[1] M. Baotić, F. J. Christophersen, and M. Morari. Constrained Optimal Control of Hybrid Systems with a Linear Performance Index. IEEE Trans. on Automatic Control, 51(12):1903-1919, December 2006.

[2] A. Bemporad and C. Filippi. Suboptimal explicit RHC via approximate multiparametric quadratic programming. Journal of Optimization Theory and Applications, 117(1):938, April 2003.

[3] A. Bemporad and M. Morari. Control of systems integrating logic, dynamics, and constraints. Automatica, 35(3):407-427, March 1999.

[4] A. Bemporad, M. Morari, V. Dua, and E. N. Pistikopoulos. The explicit linear quadratic regulator for constrained systems. Automatica, 38(1):3-20, January 2002.

[5] A. Bemporad, A. Oliveri, T. Poggi, and M. Storace. Synthesis of stabilizing model predictive controllers via canonical piecewise affine approximations. In Conference on Decision and Control, CDC, pages 5296-5301, Atlanta, USA, December 2010.

[6] F. Borrelli. Constrained Optimal Control of Linear and Hybrid Systems, volume 290 of Lecture Notes in Control and Information Sciences. Springer-Verlag, 2003.
[7] N. Brisebarre, S. Chevillard, M.D. Ercegovac, J.-M. Muller, and S. Torres. An efficient method for evaluating polynomial and rational function approximations. In Proceedings of the ASAP 2008 Conference, pages 233 -238, July 2008.

[8] F. J. Christophersen. Optimal Control of Constrained Piecewise Affine Systems, volume 359 of Lecture Notes in Control and Information Sciences. Springer Verlag, 2007.

[9] J. Eve. The Evaluation of Polynomials. Numerische Mathematik, 6:17-21, 1964.

[10] T. Geyer, F.D. Torrisi, and M. Morari. Optimal complexity reduction of polyhedral piecewise affine systems. Automatica, 44(7):1728-1740, July 2008.

[11] G. H. Hardy, J. E. Littlewood, and G. Pólya. Inequalities. Cambridge University Press, second edition, 1952.

[12] M. Hovd, F. Scibilia, J. Maciejowski, and S. Olaru. Verifying stability of approximate explicit MPC. In IEEE Conference on Decision and Control, pages 6345-635, Shanghai, China, December 2009.

[13] S. Hovland, K. E. Willcox, and J. T. Gravdahl. Explicit MPC for large-scale systems via model reduction. AIAA Journal of Guidance, Control and Dynamics, 31(4), July 2008.

[14] ILOG, Inc. CPLEX User Manual. Gentilly Cedex, France. http://www.ilog.fr/products/cplex/.

[15] T.A. Johansen and A. Grancharova. Approximate explicit constrained linear model predictive control via orthogonal search tree. IEEE Trans. on Automatic Control, 48:810-815, May 2003.

[16] M. Kvasnica, F. J. Christophersen, M. Herceg, and M. Fikar. Polynomial approximation of closed-form MPC for piecewise affine systems. In Proceedings of the 17th IFAC World Congress, pages 3877-3882, Seoul, Korea, July 6-11 2008.

[17] M. Kvasnica and M. Fikar. Performance-lossless complexity reduction in explicit MPC. In Conference on Decision and Control, CDC, pages 5270-5275, Atlanta, USA, December 2010.

[18] M. Kvasnica, P. Grieder, and M. Baotić. Multi-Parametric Toolbox (MPT), 2004. Available from http://control.ee.ethz. $\mathrm{ch} /{ }^{\sim} \mathrm{mpt} /$.

[19] M. Kvasnica, J. Löfberg, M. Herceg, L'. Čirka, and M. Fikar. Low-complexity polynomial approximation of explicit MPC via linear programming. In Proceedings of the American Control Conference, pages 4713-4718, Baltimore, USA, 2010.

[20] M. Lazar, D. Munoz de la Pena, W.P.M.H. Heemels, and T. Alamo. On input-to-state stability of min-max nonlinear model predictive control. Systems 83 Control Letters, 57:3948, 2008.

[21] J. Löfberg. YALMIP : A Toolbox for Modeling and Optimization in MATLAB. In Proc. of the CACSD Conference, Taipei, Taiwan, 2004. Available from http: //users.isy.liu.se/johanl/yalmip/.

[22] H. Mok and W. To. Effective Pólya semi-positivity for nonnegative polynomials on the simplex. Journal of Complexity, 24(4):524 - 544, 2008.

[23] P. Tøndel, T. A. Johansen, and A. Bemporad. Evaluation of Piecewise Affine Control via Binary Search Tree. Automatica, 39(5):945-950, May 2003.

[24] P. Tøndel and T.A. Johansen. Complexity reduction in explicit linear model predictive control. In Proc. of 15-th IFAC World Congress, 2002.

[25] Ch. Wen, X. Ma, and B. E. Ydstie. Analytical expression of explicit MPC solution via lattice piecewise-affine function. Automatica, 45(4):910 - 917, 2009. 